\title{
A Preliminary Study on the Characteristics of Low-speed DC Wind Tunnel and Full-flow Simulation
}

\author{
Ren Guangxu, Hua Xin, Wang Duo \\ Department of Aviation Theory, Air Force Aviation University, Changchun, 130022
}

Keywords: low speed DC wind tunnel, full flow field, simulation, turbulence model

Abstract. In this paper, the basic characteristics of fluid flow in low-speed DC wind tunnel are
described. Under the premise of clarifying the basic characteristics of low-speed DC wind tunnel, a
full-flow turbulence model is proposed. The basic method of full flow field simulation is described.

\section{Introduction}

The low-speed DC wind tunnel is a kind of pipe-like experimental equipment which can produce and control the airflow manually and simulate the flow of the fluid around the aircraft or the object and measure the effect of the airflow on the object and observe the physical phenomena. Low-speed DC wind tunnel is a basic equipment for aerodynamics experiments, so far the most aerodynamic experiments were done in a wind tunnel. Due to the complexity of the gas flow phenomenon, many of the problems in aerodynamics research can not be solved simply by theoretical or analytical methods, but must pass a large number of experiments to find out their laws or provide data and combine them with theoretical analysis, in order to solve practical problems.

Low-speed DC wind tunnel produced by a stable and uniform air flow, for aerodynamic testing is very important. The flow field quality of the wind tunnel test section directly affects the reliability of the experimental results. Therefore, the flow field quality of the wind tunnel test section should be evaluated before the aerodynamic experiment.

\section{Basic Characteristics of Flow Field in Wind Tunnel Test Section}

The structure of the low-speed DC wind tunnel is generally composed of the shrinkage current collector section, the test section, the expansion section and the axial fan. The main purpose is to test the flow field characteristics of the test section. The main indexes of the test are flow field uniformity, turbulence, airflow stability and so on.

In a variety of aerodynamic experiments, one or more of the airflow parameters (such as pressure, flow rate, temperature, flow, flow, etc.) needs to be measured. In order to reduce and eliminate systematic errors, the flow field must meet the quality standards. For this reason, it is necessary to measure the spatial distribution of airflow parameters in the empty wind tunnel (no model) before performing aerodynamic experiments, namely flow characteristics study, also called flow field calibration.

The experimental section is the place where the model is installed. The aerodynamic characteristics of the airflow in this area determine the success or failure of the wind tunnel design and manufacture, which directly affects the reliability of the measurement results. It is the final embodiment of the work of the wind tunnel. Any wind tunnel to ensure that the experimental section of the flow field uniform, turbulence is low, specifically the following requirements:

(1) Air flow parameters - flow rate, pressure, temperature, etc. in any section of the experimental section as much as possible to achieve uniform distribution, and does not change with time. The average error of the average velocity usually requires a certain value;

(2) The stability of the air flow is generally less than the specified value;

(3) The angle between the airflow direction and the wind tunnel axis should be as small as possible;

(4) It has a consistent turbulence requirement less than the specified value. 


\section{Low Speed DC Wind Tunnel Full Flow Field Simulation (FFM) Method}

The simulation methods commonly used in low - speed DC wind tunnel are zonal simulation, full flow simulation and large eddy simulation. In the full flow field simulation (FFM), the partial equations are used to describe the amount that has been averaged and the same equation is used throughout the flow. In the constant incompressible flow, the time average is used. Variables include at least velocity fields, and may also include various mean turbulence parameters such as turbulent kinetic energy, turbulent stress tensor, and so on. For compressible flows, other thermodynamic state variables must also be calculated. For periodic flow in a piston engine, the time must be averaged to ensure that the phase is averaged.

In FFM, each model of the turbulence is required for each average. These models must reflect the characteristics of turbulence at all scales. The equation for controlling the statistical properties of the flow is derived from the basic equation of the dominant density $\rho$, the velocity $u_{\mathrm{i}}$, the intrinsic energy per unit mass $e$, and the concentration $Y_{\mathrm{a}}$ of per unit mass. These equations can be written in the most important way:

$$
\begin{gathered}
\frac{D}{D t}\left(\begin{array}{l}
1 \\
u_{i} \\
e \\
Y a
\end{array}\right)=\left(\begin{array}{l}
0 \\
0 \\
Q \\
S a
\end{array}\right)-\frac{\partial}{\partial x_{j}}\left(\begin{array}{l}
0 \\
\tau_{i j} \\
q_{j} \\
J a j
\end{array}\right) \\
\frac{D f}{D t} \equiv \frac{\partial(\rho f)}{\partial t}+\frac{\partial}{\partial x_{j}}\left(\rho u_{j} f\right)
\end{gathered}
$$

Where $D / D t$ is the mass convection operator.

The thermal energy item $Q$ includes a viscosity term and a source term caused by a chemical reaction. Both the $Q$ and the material source $S_{\mathrm{a}}$ will depend on the chemical reaction rate equation, which must be known to solve the problem. The thermodynamic equation of state is also needed. In addition, the structural equation for the diffusion flux must be specified.

Studies have shown that, in addition to the thermal diffusion of conduction, the diffusion of various substances may also form part of the diffusion stream $q_{\mathrm{j}}$ of the internal energy.

In the FFM method, follow the equation (1) for the average variable. Most turbulence models are developed with time averages.

Analysis of the flow in the low-speed DC wind tunnel requires consideration of compressibility. One way to do this is to apply simple phase averages from beginning to end. The other is the mass weighted average, which makes the average compressible flow equation appear exactly the same as the average incompressible flow equation. In this way, the law of the average equation does not have additional items that the new model must add. Thus, a model that is very successful for incompressible flow can be directly extended to the compressible flow equation. Of course, this promotion is pure mathematics, from the physical confirmation of this promotion need to be verified by experiment.

The composite phase averaging method is the basis for analyzing the flow of the engine and is therefore described in detail here. We will use brackets to represent the phase averaging process:

$$
\{\rho(x, t)\}=\lim _{N \rightarrow \infty} \frac{1}{N} \sum_{n=1}^{n} \rho(x, t+n \tau)
$$

Where $\tau$ is a period of the cycle, we also expressed as $\{\rho\}=\tilde{\rho}$. Then $\rho$ is decomposed into $\rho=\tilde{\rho}+\rho^{\prime}$. The weight-weighted phase averaging process is expressed by means of brackets and the mass-weighted average of phase represented by a dash on top, which is

$$
\tilde{\rho}(\underline{x}, t)<f(\underline{x}, t)>=\lim _{N \rightarrow \infty}\left[\frac{1}{N} \sum_{n=1}^{N} \rho(\underline{x}, t+n \tau) \bar{f}(\underline{x}, t+n \tau)\right]=\tilde{\rho} \bar{f}
$$

We put all the flow variables (except density and pressure) into $f=\tilde{f}+f^{\prime}$. Equation (1) is averaged to obtain: 


$$
\bar{D} \overline{D t}\left(\begin{array}{l}
1 \\
\bar{u}_{i} \\
\bar{e} \\
\overline{Y a}
\end{array}\right)=\left(\begin{array}{l}
0 \\
0 \\
\{\bar{Q}\} \\
\{S a\}
\end{array}\right)-\frac{\partial}{\partial x_{j}}\left(\begin{array}{l}
0 \\
\left\{\tau i_{j}\right\} \\
\left\{q_{j}\right\} \\
\left\{J_{a j}\right\}
\end{array}\right)-\frac{\partial}{\partial x j}\left(\begin{array}{l}
0 \\
\tilde{\rho} u_{i}^{\prime} u_{j}^{\prime} \\
\tilde{\rho} e^{\prime} u_{j}^{\prime} \\
\tilde{\rho} Y_{a} u_{j}^{\prime}
\end{array}\right)
$$

Where

$$
\frac{\bar{D} \bar{f}}{D t} \equiv \frac{\partial}{\partial t}(\tilde{\rho} \bar{f})+\frac{\partial}{\partial x_{j}}\left(\tilde{\rho} \bar{u}_{j} \bar{f}\right)
$$

The left side of Equation (5) contains only the variables $\tilde{\rho} 、 \bar{u}_{i} 、 \bar{e}$ and $\overline{Y a}$, and therefore no model. However, all the items on the right, especially the last items representing the turbulent transport, contain turbulence pulsations, and the model must be modeled with the variable expression.

The momentum equation contains the term $-\tilde{\rho} \bar{u}_{i}^{\prime} \vec{u}_{j}^{\prime}$, which represents the turbulence stress. In the stress equation simulation, the equations of these stresses are listed by the model and can be solved. Add two partial differential equations describing the velocity and scale of the turbulence to equation (5), and then express the other turbulence with these scales.

The equation of the turbulence model utilizes the equations of the turbulence kinetic energy $k=\bar{u}_{i} \bar{u}_{j} / 2$ in the control unit mass. The equation for controlling $\mathrm{k}$ can be derived by multiplying the ui in equation (1) by uj, subtracting $\bar{u}_{i}$ obtained by from equation (5) multiplying $\bar{u}_{i}$, and then averaging the result. The results find the k equation as:

$$
\frac{\bar{D} \bar{k}}{D t}=\tilde{\rho}(p-e)-\frac{\partial}{\partial x_{j}} J_{k}
$$

Where $p$ is the turbulence generation rate per unit mass:

$$
p=-\bar{u}_{i} \bar{u}_{j} \frac{\partial \bar{u}_{i}}{\partial x_{j}}
$$

In the actual experimental verification, all the equations are used as a second turbulence equation using a dissipative model equation. Since the energy dissipation rate is not controlled by the viscosity and is controlled by the rate at which the large eddy energy is supplied to the small dissipation scale, the latter itself adjusts the size to handle such energy. So, if $l$ is large vortex turbulence length scale, dissipation should be fixed on $\mathrm{k}$ and $l$ :

$$
e \propto k^{2 / 3} / l
$$

In the turbulence model, all unknown turbulence is expressed by the turbulence velocity scale $k^{1 / 2}$ and the length $k^{2 / 3} / \mathrm{e}$, or $k$ and $e$. Thus, this model is called the $k$-e model. The general method involves defining a turbulent viscosity:

$$
\mu_{T} \equiv \operatorname{Co} \tilde{\rho} k^{2} / e
$$

Then the turbulent stress term appearing in equations (5) and (7) is simulated by quasi-Newtonian method:

$$
\tilde{\rho} \bar{u}_{i}^{\prime} \vec{u}_{j}^{\prime}=\tilde{\rho} \frac{2}{3} k \delta_{i j}+\frac{2}{3} \mu_{T} \nabla \bar{u} \delta_{i j}-2 \mu_{T} \bar{S}_{i j}
$$

Where $\bar{s}_{i j}$ is the strain rate of the field $\bar{u}_{i}$ :

$$
\bar{S}_{i j}=\frac{1}{2}\left(\frac{\partial \bar{u}_{i}}{\partial x_{j}}+\frac{\partial \bar{u}_{j}}{\partial x_{i}}\right)
$$

The viscous stress term in the momentum equation is estimated by the Newtonian structural equation. They include additional turbulence associated with pulsations of molecular viscosity.

The turbulence diffusion term in the various transport equations is simulated by the turbulence diffusion coefficient. The diffusion flux of $\phi$ is simulated by the following equation: 


$$
J_{\Phi i}=-\frac{\mu_{T}}{\delta_{\phi}} \cdot \frac{\partial \phi}{\partial x_{i}}
$$

Finally, the model is done with an e-transport equation. The exact equation of e can be derived from the proper treatment of the $N$-S equation, which is a useful guide for building the e-equation. All e-equation models have this form:

$$
\frac{\bar{D} e}{D t}=W-\frac{\partial H_{i}}{\partial x_{i}}
$$

The diffusion flux $H_{\mathrm{i}}$ in the equation $\bar{D} e$ is simulated in the same way as other diffusion terms.

The above equation model has been successfully applied to free and wall-constrained shear layers. With the above modifications, this model can be well simulated even in areas close to the wall. However, if you want to make the calculation to the wall, it is need to use a very fine calculation of the grid, so the usual procedure is to dissolve near the wall to fit a hypothetical "universal" wall form. However, in the engine, the wall boundary layer does not have time to relax to the local equilibrium state, so this wall treatment is a potential source of error.

\section{Conclusion}

After the above analysis, the flow structure can be predicted from the study of the flow model, and the turbulence parameter value in the simple low-speed DC wind tunnel should be given. In a layered system, a good flow model should be able to predict the charge distribution at the shrinkage current collector. If you can build a model to deal with the flow process, then you should at least predict the pressure over time and estimate the flow of the situation. If the model can properly handle the circulation in the flow, it is also possible to predict the time course of the end of the test.

The most useful type of experiment for the study model is that there is only one factor change and other unchanging. Other basic experiments include experiments on unsteady compressible boundary layers, experiments in unsteady flow fields in compressible flows, and experiments involving only flowing from a very complete turbulence field.

It is worth noting that the verification of the model should rely on the actual low-speed DC wind tunnel experiments to record the flow field, pressure and exhaust composition changes over time. Such experiments should be based on changes in the system parameters, predictive analysis model with the design parameters of the trends and laws.

\section{References}

[1] R. J. Tabaczynski, "Turbulence and Turbulent Combustion in Spark-Ignition Engincs." Prog. Energy Combust. Sci. Vol. 2. pp. 143-165, 1976

[2] W.C.Reynoynolds. Simulation of Internal Combustion Engine Combustion. Stanford University. Liu Yijun translation. 1987.8

[3] Weekly line. Combustion chamber modeling and efficiency analysis of mist liquid fuel. Gas turbine combustor test technology special academic conference papers anthology. Shanghai Mechanical Engineering Society. Beijing: China Industry Press, 1964

[4] Chen Danzhi. The Modes of Steady State of Low - speed Combustion System. Journal of Engineering Thermophysics, 1980,1 (4)

[5] Liu Qingguo. Experimental Study on Combustion Chamber Performance of Pneumatic Atomization Nozzle. Combustion Science and Technology, 2nd Series, 1984

[6] China National People's Liberation Army General Assembly military training materials editorial work committee high and low speed wind tunnel pneumatic and structural design [M] Beijing: National Defense Industry Press, 2003 